\title{
ON ABSOLUTE MATRIX SUMMABILITY FACTORS OF INFINITE SERIES
}

\author{
AHMET KARAKAŞ
}

Abstract. In the present paper, a general theorem dealing with $\left|A, p_{n} ; \delta\right|_{k}$ summability method of infinite series has been proved by using almost increasing sequences. Some results have also been given.

Mathematics subject classification (2010): 26D15, 40D15, 40F05, $40 \mathrm{G} 99$.

Keywords and phrases: Absolute matrix summability, almost increasing sequences, Hölder inequality, infinite series, Minkowski inequality, summability factor.

\section{REFERENCES}

[1] N. K. BARI AND S. B. SteČKIn, Best approximations and differential properties of two conjugate functions, Trudy. Moskov. Mat. Obšč. 5 (1956), 483-522 (in Russian).

[2] H. Bor, On two summability methods, Math. Proc. Cambridge Philos. Soc. 97 (1985), 147-149.

[3] H. BoR, A note on $\left|\bar{N}, p_{n}\right|_{k}$ summability factors of infinite series, Indian J. Pure Appl. Math. 18 (1987), 330-336.

[4] H. Bor, On absolute Riesz summability factors, Adv. Stud. Contemp. Math. (Pusan), 3 (2) (2001), 23-29.

[5] H. Bor, A note on absolute Riesz summability factors, Math. Inequal. Appl. 10 (2007), 619-625.

[6] H. BoR, A new application of almost increasing sequences, J. Comput. Anal. Appl. 10 (2008), $17-23$.

[7] T. M. FLETT, On an extension of absolute summability and some theorems of Littlewood and Paley, Proc. London Math. Soc. 7 (1957), 113-141.

[8] S. M. Mazhar, A note on absolute summability factors, Bull. Inst. Math. Acad. Sinica 25 (1997), 233-242.

[9] K. N. Mishra AND R. S. L. SRIVAstava, On $\left|\bar{N}, p_{n}\right|$ summability factors of infinite series, Indian J. Pure Appl. Math. 15 (1984), 651-656.

[10] H. S. ÖZARSLAN AND H. N. ÖĞDÜK, Generalizations of two theorems on absolute summability methods, Aust. J. Math. Anal. Appl. 1 (2004), Article 13, 7 pp.

[11] H. S. ÖZARsLan, A new application of almost increasing sequences, Miskolc Math. Notes. 14 (2013), 201-208.

[12] H. S. ÖZARSLAn, On generalized absolute matrix summability, Asia Pacific J. Math. 1 (2) (2014), $150-156$.

[13] H. S. Özarslan, A new application of absolute matrix summability, C. R. Acad. Bulgare Sci. 68 (2015), 967-972.

[14] H. S. ÖZarslan, A new study on generalized absolute matrix summability, Commun. Math. Appl. 4 (2016), 303-309.

[15] H. S. ÖZarslan, A new application of generalized almost increasing sequences, Bull. Math. Anal. Appl. 8 (2016), 9-15.

[16] H. S. ÖZARSLAN AND A. KARAKAŞ, A new result on the almost increasing sequences, J. Comp. Anal. Appl. 22 (2017), 989-998.

[17] H. S. ÖZArslan And B. Kartal, A generalization of a theorem of Bor, J. Inequal. Appl. 179 (2017), 1-8.

[18] W. T. Sulaiman, Inclusion theorems for absolute matrix summability methods of an infinite series. IV, Indian J. Pure Appl. Math. 34 (11) (2003), 1547-1557. 